\title{
Replication of the association of the aspartic acid repeat polymorphism in the asporin gene with knee-osteoarthritis susceptibility in Han Chinese
}

\author{
Qing Jiang $\cdot$ Dongquan Shi $\cdot$ Long Yi $\cdot$ Shiro Ikegawa $\cdot$ Yong Wang $\cdot$ \\ Takahiro Nakamura $\cdot$ Di Qiao $\cdot$ Cheng Liu $\cdot$ Jin Dai
}

Received: 10 August 2006/ Accepted: 28 August 2006/ Published online: 21 September 2006

(C) The Japan Society of Human Genetics and Springer 2006

\begin{abstract}
A genetic association of osteoarthritis (OA) and functional polymorphisms in the aspartic acid (D) repeat of the asporin gene was reported in Japanese and European Caucasians; however, the results were controversial. Our objective was to evaluate whether the $\mathrm{D}$ repeat polymorphism was associated with knee $\mathrm{OA}$ in Han Chinese. The D repeat polymorphism was genotyped in 218 patients who suffered from primary symptomatic knee OA with radiographic confirmation and in 454 age-matched controls, and the allelic association of the repeat was examined. Frequencies of the D13 and D14 alleles were similar to those of Japanese, but different from those of European Caucasians. The
\end{abstract}

Electronic supplementary material Supplementary material is available in the online version of this article at http://dx.doi.org/ 10.1007/s10038-006-0065-6 and is accessible for authorized users.

Q. Jiang $(\varangle) \cdot$ D. Shi $\cdot$ C. Liu $\cdot$ J. Dai

The Center of Diagnosis and Treatment for Joint Disease,

Drum Tower Hospital Affiliated to Medical School

of Nanjing University, Zhongshan Road 321,

Nanjing 210008, Jiangsu, China

e-mail: qingj@nju.edu.cn

L. Yi · Y. Wang · D. Qiao

Department of Pathology,

Medical School of Nanjing University,

Nanjing, China

S. Ikegawa

Laboratory for Bone and Joint Diseases,

SNP Research Center, RIKEN, Tokyo, Japan

T. Nakamura

Laboratory for Statistical Analysis,

SNP Research Center, RIKEN, Tokyo, Japan
D14 allele was significantly over-represented in knee OA patients $(P=0.0013$; odds ratio 2.04 ; $95 \%$ confidence interval 1.32-3.15). D14 was more frequent in early-onset patients than in late-onset patients $(P=0.043)$ and the age at onset in patients with D14 was earlier $(P=0.028$; log-rank test $)$. Thus, the association of the D14 allele with knee OA susceptibility was replicated in Han Chinese. This was the first instance that association of the OA susceptibility gene was definitely replicated between different ethnic groups.

Keywords Asporin - Microsatellite polymorphism . Osteoarthritis · Han Chinese · Case control

\section{Introduction}

Osteoarthritis (OA, OMIM 165720) is one of the most common skeletal diseases characterized by the progressive loss of articular cartilage in synovial joints. $\mathrm{OA}$ is the most common cause of the limitation of activities of daily living after middle age. Knee OA, in particular, has a high prevalence in Asia; recent studies in China show that the prevalence of radiographic and symptomatic knee OA among women aged 60 and over were 42.8 and $15.4 \%$, respectively (Zhang et al. 2004). Epidemiological studies have shown that OA has a strong genetic component, and several susceptibility genes for OA have been reported (Loughlin 2005).

Recent genetic studies have focused on $A S P N$, the gene encoding asporin. Asporin is a new member of the small leucin-rich proteoglycan family (Lorenzo et al. 2001; Henry et al. 2001). Asporin has consecutive aspartic acid residues (D repeat) in the amino-terminal 
region of its mature protein determined by a microsatellite polymorphism. Kizawa et al. (2005) first reported a strong association of $A S P N$ with knee and hip $\mathrm{OA}$ in the Japanese. Together with convincing functional evidence, they show that the D14 allele (an allele containing $14 \mathrm{D}$ repeats) is over-represented, and the D13 allele is under-represented in OA. However, the association has not been replicated clearly in subsequent studies in European Caucasians (Mustafa et al. 2005; Kaliakatsos et al. 2006; Rodriguez-Lopez et al. 2006). In the UK study (Mustafa et al. 2005), there is a significant association of the D14 allele in hip OA, but only in males. A tendency is observed in the same sense as in the Japanese study: an increase of the D14 allele and a decrease of the D13 allele in knee and hip OA. In knee OA of the Greek population, a significant decrease of the D13 allele and increased frequencies of the D15 and D18 alleles are found in knee OA, but there is no association in the D14 allele (Kaliakatsos et al. 2006). The Spanish study has detected no association in knee, hip and hand OA in any comparisons and stratifications (Rodriguez-Lopez et al. 2006). However, by reviewing the literature, Ikegawa et al. (2006) suggested that the combined result of Europeans is positive for the association of the D14 allele and knee OA.

The association of asporin and OA seems promising, but thus controversial. To clarify its global relevance, the association has to be confirmed by independent studies in different ethnic groups. The purpose of the present study is to examine the genetic association of the $\mathrm{D}$ repeat polymorphism in asporin with knee OA in a Han Chinese population.

\section{Patients and methods}

\section{Subjects and protocol}

A total of 672 subjects were studied. Two hundred eighteen patients (151 women and 67 men) were enrolled consecutively at the Center of Diagnosis and Treatment for Joint Disease, Drum Tower Hospital, affiliated with the Medical School of Nanjing University; 454 healthy control subjects (289 women and 165 men) were enrolled at the Center of Physical Examination. The control group had never had any signs or symptoms of arthritis or joint diseases (pain, swelling, tenderness, or restriction of movement). All subjects included in the study were Han Chinese living in and around Nanjing. No subjects dropped out during the process of the study. The study was approved by the ethical committee of the Medical School of Nanjing
University, and informed consent was obtained from patients and controls.

Knee OA patients who had not only definite signs and symptoms of $\mathrm{OA}$, but also radiographic evidence of OA were included. All the patients had pain with rest and/or night pain of over 5-month duration. Other etiologies causing knee diseases such as inflammatory arthritis (rheumatoid, polyarthritic or autoimmune disease), posttraumatic or postseptic arthritis, skeletal dysplasia or developmental dysplasia were excluded. Radiographic OA was assessed using the Kellgren/ Lawrence (K/L) grading system (Kellgren and Lawrence 1963). Only patients with K/L grades of 2 or higher were included. To all subjects, we calculated the body mass index (BMI; body weight in kilograms divided by the square of the height in meters) to assess obesity. The age of onset was investigated during the later period of the study.

\section{Genotyping}

Genomic DNA was extracted from peripheral blood leukocytes using the Chelex-100 method (Walsh et al. 1991) or from buccal swabs using the DNA IQ System (Promega, Madison, WI) according to the manufacturer's instructions. DNA was genotyped for the $A S P N$ microsatellite encoding the D repeat polymorphism after PCR amplification using $50 \mathrm{ng}$ of genomic DNA and primers designed by online Primer 3 Software (http://www.cbr-rbc.nrc-cnrc.gc.ca/cgi-bin/primer3_ www.cgi), 5'-CCC TTC TTT AGC CCT TCA CAC-3' (forward) and 5'-CAC TGA CAT CCA AAT GGA CAC-3' (reverse). PCR was performed in a PTC-100 thermal-cycler (MJ Research, USA) as follows: 30 cycles consisting of $1 \mathrm{~min}$ of denaturation at $94^{\circ} \mathrm{C}$, $1 \mathrm{~min}$ of annealing at $60^{\circ} \mathrm{C}$ and $1 \mathrm{~min}$ of extension at $72^{\circ} \mathrm{C}$ with an initial denaturation step of 5 min at $94^{\circ} \mathrm{C}$ and a final extension of $10 \mathrm{~min}$ at $72^{\circ} \mathrm{C}$. PCR products with $2 \mu \mathrm{l}$ STR $2 \times$ Loading Solution (Promega) were loaded onto $6 \%$ denaturing polyacrylamide gel (BIORAD Sequi-Gen GT System $38 \times 30 \mathrm{~cm}$, CAT. no. 165 3862). Samples were run at $50^{\circ} \mathrm{C}$ for about $2 \mathrm{~h}$. After electrophoresis, the gels were stained with silver nitrate. Allele size determination was carried out by comparison to an allele ladder.

\section{Statistical analysis}

Fisher's exact test was used to compare the $A S P N$ allele distributions in the case-control study. Stratification analysis by age, sex, BMI and $\mathrm{K} / \mathrm{L}$ scores was performed using SPSS 12.0 system software. We assessed the association of D14 with the stratifications 
and the Hardy-Weinberg equilibrium by the $\chi^{2}$ test. Odds ratio (OR), $\mathrm{P}$ value and $95 \%$ confidence interval (CI) were calculated with respect to the minor allele compared with the major allele. The stratification analyses of the age at onset were performed using SPSS 12.0 and statistical software R. We tested the difference of the age at onset of OA between the D13 and D14 genotypes using Mann-Whitney and KruskalWallis tests. We also applied a survival analysis using the Kaplan-Meier method and log-rank test for the date.

\section{Results}

The ages of the patients and the controls (mean \pm SD) were $58.1 \pm 18.9$ (range 32-89) years and 56.3 \pm 12.1 (range 40-97) years, respectively. BMI of the patients and the controls (mean $\pm \mathrm{SD}$ ) were 25.3 \pm 3.64 and $23.4 \pm 3.83 \mathrm{~kg} / \mathrm{m}^{2}$. There was no statistical difference between the two groups. Over $70 \%$ of the patients had a K/L score of 3 or 4 . Eighty-five patients had an earlyonset age (onset age $<50$ years), while 107 patients had a late-onset age (onset age $>50$ years). Eight different alleles were identified, corresponding to $11-18 \mathrm{D}$ repeats (Table 1). There were 17 genotypes. Distributions of genotypes in the knee OA and control groups were conformed to Hardy-Weinberg equilibrium ( $P=0.781$ and $P=0.293$, respectively). Distribution of allelic frequencies was extraordinarily similar between Han Chinese and Japanese. However, a significant difference was detected between Han Chinese and UK Caucasians $(P=0.0006)$. As in the Japanese and the European populations (Kizawa et al. 2005; Mustafa et al. 2005; Kaliakatsos et al. 2006; Rodriguez-Lopez et al. 2006), the most common allele was D13 in both patients and controls. The allelic frequency of D13 in Han Chinese was similar to that in Japanese and much higher than that in European Caucasians. The allele frequency of D14 was also similar to that in Japanese and much lower than that in European Caucasians.

A significant difference in the allelic frequency was observed in a comparison of D14 versus other alleles combined $(P=0.0013$; Table 2$)$, but was found in a comparison of D12 versus other alleles combined $(P=0.004)$. The association can overcome the Bonferroni's correction of multiple testing for the number of alleles tested (corrected $P: 0.05 / 8=0.0063$ ). The association was also found after stratification by sex (female, $P=0.024 ;$ male, $P=0.019)$. No significant association was detected for D13 versus other alleles combined in any comparisons. Also, no significant differences were observed in any other alleles including D15 and D18 for comparisons of one allele versus

Table 1 Allelic frequency of the aspartic acid (D)-repeat polymorphism of asporin in knee osteoarthritis (KOA) in a Han Chinese population

\begin{tabular}{|c|c|c|c|c|c|c|c|c|c|c|}
\hline \multirow[t]{2}{*}{ Group } & \multirow{2}{*}{$\begin{array}{l}\text { Number of } \\
\text { subject }\end{array}$} & \multicolumn{9}{|c|}{ Number of allele (\%) } \\
\hline & & D11 & D12 & D13 & D14 & D15 & D16 & D17 & D18 & Total \\
\hline \multicolumn{11}{|l|}{ KOA } \\
\hline All & 218 & $3(6.8)$ & $61(14.0)$ & $300(68.8)$ & $41(9.4)$ & $11(2.5)$ & $15(3.4)$ & $5(1.1)$ & 0 & 436 \\
\hline Female & 151 & $3(0.1)$ & $44(14.5)$ & $208(68.8)$ & $29(9.6)$ & $4(1.3)$ & $11(3.6)$ & $3(0.1)$ & 0 & 302 \\
\hline Male & 67 & 0 & $17(12.7)$ & $92(68.6)$ & $12(9.0)$ & $7(5.2)$ & $4(3.0)$ & $2(1.5)$ & 0 & 134 \\
\hline \multicolumn{11}{|c|}{ Non-KOA } \\
\hline All & 454 & 0 & $186(20.5)$ & $604(66.5)$ & $44(4.9)$ & $29(3.2)$ & $39(4.3)$ & $3(0.3)$ & $3(0.3)$ & 908 \\
\hline Female & 289 & 0 & 113 (19.5) & $387(67.0)$ & $32(5.5)$ & $16(2.8)$ & $28(4.8)$ & $1(0.2)$ & $1(0.2)$ & 578 \\
\hline Male & 165 & 0 & $73(22.1)$ & $217(65.8)$ & $12(3.6)$ & $13(4.0)$ & $11(3.3)$ & $2(0.6)$ & $2(0.6)$ & 330 \\
\hline
\end{tabular}

Table 2 Association of the D-repeat of asporin in patients with knee osteoarthritis in a Han Chinese population

\begin{tabular}{|c|c|c|c|c|c|c|c|c|c|}
\hline \multirow[t]{2}{*}{ Groups compared } & \multicolumn{3}{|c|}{ D14 vs. others } & \multicolumn{3}{|c|}{ D13 vs. others } & \multicolumn{3}{|c|}{ D14 vs. D13 } \\
\hline & OR & $P$ value & $95 \% \mathrm{CI}$ & OR & $P$ value & $95 \% \mathrm{CI}$ & OR & $P$ value & $95 \% \mathrm{CI}$ \\
\hline $\begin{array}{l}\text { All patients }(n=218) \text { versus } \\
\text { all controls }(n=454)\end{array}$ & 2.04 & 0.0013 & $1.32-3.15$ & 1.11 & 0.40 & $0.87-1.42$ & 1.88 & 0.0052 & $1.21-2.92$ \\
\hline $\begin{array}{l}\text { Female patients }(n=151) \text { versus } \\
\quad \text { female controls }(n=289)\end{array}$ & 1.81 & 0.024 & $1.08-3.04$ & 1.09 & 0.56 & $0.81-1.47$ & 1.69 & 0.05 & $1.00-2.85$ \\
\hline $\begin{array}{l}\text { Male patients }(n=67) \text { versus } \\
\text { male controls }(n=165)\end{array}$ & 2.61 & 0.019 & $1.17-5.81$ & 1.14 & 0.55 & $0.74-1.75$ & 2.36 & 0.039 & $1.04-5.34$ \\
\hline
\end{tabular}


all the remaining alleles combined. A Significant difference was observed in D14 versus D13 $(P=0.0052)$.

There were no significant differences in the allelic frequencies of D13 and D14 between age and BMI. There was no correlation between the allelic frequencies of D14 and severity of OA (K/L scores). D14 was more frequent in early-onset patients than in late-onset patients $(P=0.043)$. The age at onset in patients with D14 (47.7 \pm 11.4 years) was younger than that in patients without D14 (52.9 \pm 11.7 years) $(P=0.016$, Mann-Whitney test). A survival analysis using the Kaplan-Meier method (supplementary Fig. 1) and the log-rank test showed a significant difference in a dominant model (with vs. without D14) $(P=0.0028)$.

\section{Discussion}

We have replicated the association of asporin with knee OA in a Han Chinese population. This is the first time that the association of OA has been replicated between different ethnic populations. The allelic frequency and OR of D14 are very similar in Han Chinese and Japanese. Our result indicates that D14 is a common susceptibility allele for knee OA at least in the East Asian population. The Greek study suggested that D15 and D18 alleles could be risk alleles (Kaliakatsos et al. 2006), but the association was not replicated in our population. We also detected that the D12 allele had a lower frequency compared with other alleles combined. The functional properties of the D12 allele have not yet been examined, and it remains to be shown if they encoded OA protection. D14 frequency is significantly increased in patients with early-onset, and the age at onset of knee OA in patients with D14 was earlier. The association of the allele and the onset of the disease need to be confirmed by further studies.

Our results have again highlighted the discrepancy of association of asporin between the Asian and Caucasians. The difference in the ascertainment criteria (Ikegawa et al. 2006) is unlikely to account for the discrepancy. Inclusion criteria are common in that all studies recruited cases of symptomatic OA with radiographic evidence. Radiographic criteria are also similar. All patients are of $\mathrm{K} / \mathrm{L}$ grade 2 or more. The degree of severity of OA is different; the European cases contain more terminal OA $(\mathrm{K} / \mathrm{L}$ grade $>2)$ than the Japanese and our cases. However, the difference in severity of OA is also an unlikely explanation. The Japanese study reported the positive correlation between the allelic frequency of D14 and the radiographic severity of OA (Kizawa et al. 2005). Then, the European cases would come to be more favorable for the positive association. In addition, our result shows no correlation between the allelic frequency of D14 and radiographic severity.

We think the ethnic difference is a more likely explanation. The allele distribution of the $\mathrm{D}$ repeat is different among the studies. We have identified eight alleles in the Chinese Han population, whereas the previous studies found up to 12 alleles (Kizawa et al. 2005; Mustafa et al. 2005; Kaliakatsos et al. 2006; Rodriguez-Lopez et al. 2006). Allele frequencies of D13 and D14 are very similar between Chinese and Japanese, but different from European Caucasians. Populations from neighboring regions typically share more recent common ancestors. Therefore, their allele frequencies are more highly correlated, a pattern that is commonly manifested as a cline of allele frequencies (Bamshad et al 2004). HapMap studies have clearly shown that there are many similarities of genome between the Han Chinese and Japanese populations (International HapMap Consortium 2005). Asporin may be a "Mongoloid" gene for OA.

The discrepancy between Asians and European Caucasians could be multi-factorial, involving differences in disease phenotype, in lifestyle and in environmental and genetic factors determined by different sets of other susceptibility genes. Further studies are necessary to test for more global relevance of asporin and to clarify the complex, heterogeneous nature of genetic susceptibility of OA.

Acknowledgment This work is supported by the National Nature Science Foundation of China (30571874).

\section{References}

Bamshad M, Wooding S, Salisbury BA, Stephens JC (2004) Deconstructing the relationship between genetics and race. Nat Rev Genet 5:598-609

Henry SP, Takanosu M, Boyd TC, Mayne PM, Eberspaecher H, Zhou W, de Crombrugghe B, Hook M, Mayne R (2001) Expression pattern and gene characterization of asporin. A newly discovered member of the leucine-rich repeat protein family. J Biol Chem 276:12212-12221

Ikegawa S, Kawamura S, Takahashi A, Nakamura T, Kamatani N (2006) Replication of association of the D-repeat polymorphism in asporin with osteoarthristis. Arthritis Res Ther 8:403 (Epub ahead of print)

International HapMap Consortium (2005) A haplotype map of the human genome. Nature 437:1299-1320

Kaliakatsos M, Tzetis M, Kanavakis E, Fytili P, Chouliaras G, Karachalios T, Malizos K, Tsezou A (2006) Asporin and knee osteoarthritis in patients of Greek origin. Osteoarthritis Cartilage 14:609-611

Kellgren JH, Lawrence JS (1963) Radiological assessment of osteoarthrosis. Ann Rheum Dis 22:237-255

Kizawa H, Kou I, Iida A, Sudo A, Miyamoto Y, Fukuda A, Mabuchi A, Kotani A, Kawakami A, Yamamoto S, Uchida 
A, Nakamura K, Notoya K, Nakamura Y, Ikegawa S (2005) An aspartic acid repeat polymorphism in asporin inhibits chondrogenesis and increases susceptibility to osteoarthritis. Nat Genet 37:138-144

Lorenzo P, Aspberg A, Onnerfjord P, Bayliss MT, Neame PJ, Heinegard D (2001) Identification and characterization of asporin. A novel member of the leucine-rich repeat protein family closely related to decorin and biglycan. J Biol Chem 276:12201-12211

Loughlin J (2005) The genetic epidemiology of human primary osteoarthritis: current status. Expert Rev Mol Med 7:1-12

Mustafa Z, Dowling B, Chapman K, Sinsheimer JS, Carr A, Loughlin J (2005) Investigating the aspartic acid (D) repeat of asporin as a risk factor for osteoarthritis in a UK Caucasian population. Arthritis Rheum 52:3502-3506
Rodriguez-Lopez J, Pombo-Suarez M, Liz M, Gomez-Reino JJ, Gonzalez A (2006) Lack of association of a variable number of aspartic acid residues in the asporin gene with osteoarthritis susceptibility: case-control studies in Spanish Caucasians. Arthritis Res Ther 8:R55

Walsh PS, Metzger DA, Higuchi R (1991) Chelex 100 as a medium for simple extraction of DNA for PCR-based typing from forensic material. Biotechniques 10:506-513

Zhang Y, Hunter DJ, Nevitt MC, Xu L, Niu J, Lui LY, Yu W, Aliabadi P, Felson DT (2004) Association of squatting with increased prevalence of radiographic tibiofemoral knee osteoarthritis. Arthritis Rheum 50:1187-1192 